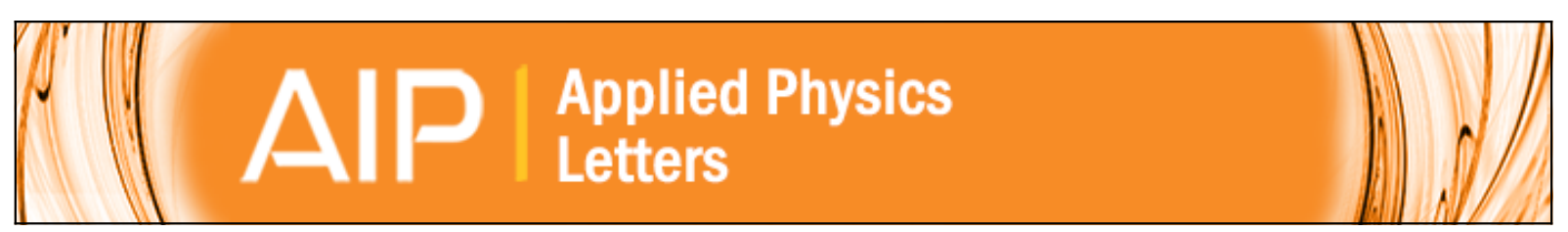

Speed enhancement of multi-particle chain in a traveling standing wave

Martin Šiler, Tomáš ižmár, and Pavel Zemánek

Citation: Applied Physics Letters 100, 051103 (2012); doi: 10.1063/1.3680234

View online: http://dx.doi.org/10.1063/1.3680234

View Table of Contents: http://scitation.aip.org/content/aip/journal/apl/100/5?ver=pdfcov

Published by the AIP Publishing

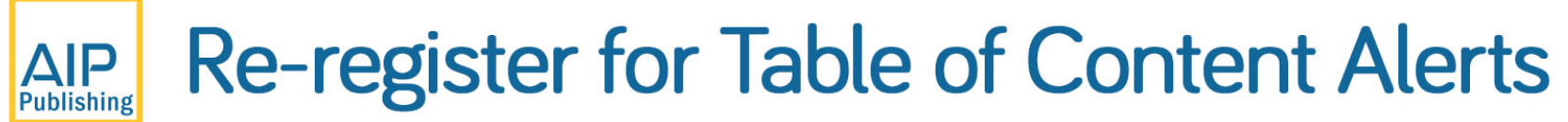

Create a profile.

Sign up today! 


\title{
Speed enhancement of multi-particle chain in a traveling standing wave
}

\author{
Martin Šiler, ${ }^{1, a)}$ Tomáš Čižmár, ${ }^{2, b)}$ and Pavel Zemánek ${ }^{1, c)}$ \\ ${ }^{1}$ Institute of Scientific Instruments of the ASCR, Královopolská 147, 61264 Brno, Czech Republic \\ ${ }^{2}$ School of Medicine, University of St. Andrews, North Haugh, St. Andrews, Fife, KY16 9TF, Scotland
}

(Received 9 December 2011; accepted 6 January 2012; published online 31 January 2012)

\begin{abstract}
A moving array of optical traps created by interference of two counter-propagating evanescent waves has been used for delivery of particle chains up to 18 micro-particles long immersed in water. The particles were optically self-arranged into a linear chain with well-separated distances between them. We observed a significant increase in the delivery speed of the whole structure as the number of particles in the chain increased. This could provide faster sample delivery in microfluidic systems. We quantified the contributions to the speed enhancement caused by the optical and hydrodynamic interactions between the particles. (c) 2012 American Institute of Physics. [doi:10.1063/1.3680234]
\end{abstract}

Optical manipulation with microparticles and nanoparticles is a well-established technique that has found numerous practical applications in a variety fields. Usually it is assumed that one or more particles are trapped in a static fluid and manipulated by repositioning of the focused trapping beams (so-called optical tweezers ${ }^{1}$ ). More advanced applications, such as optical sorting ${ }^{2-5}$ or delivery, ${ }^{6}$ use spatially structured beams to influence particles behavior in static or dynamic fluids. In the case where multiple particles are simultaneously trapped a mutual interaction between the particles takes place. This interaction is also called optical binding $^{7}$ and modifies the particles' equilibrium positions or can even create self-arranged structures of the particles. ${ }^{8-13}$ In the case where multiple illuminated particles are in motion within the fluid, the hydrodynamic interactions take place which make the inter-particle interactions even more complex. ${ }^{14}$ The hydrodynamic interactions between multiple particles have been also studied with the optical manipulation techniques for particles arranged into linear chains, ${ }^{15,16}$ ring-like structures, ${ }^{17-20}$ or two-dimensional matrix structures. ${ }^{18}$ However, most of these approaches used a set of independent optical traps and studied the hydrodynamic interactions through their coupled Brownian motion.

Utilization of light forces for particle delivery over an extended range was demonstrated near a surface ${ }^{4,6}$ or optical waveguide $^{21}$ and in bulk liquid medium using counterpropagating beams. ${ }^{22-24}$ Moreover, Grujic et al. ${ }^{25}$ reported that a pair of particles, a so called bi-particle, moved along a planar waveguide $15 \%$ faster compared to a single particle.

In this paper we focus on the behavior of a linear chain of polystyrene micro-particles that are optically selfarranged in an evanescent standing wave. ${ }^{26} \mathrm{~A}$ constant phase shift of one beam results in a constant velocity $u$ of the standing wave interference fringes and consequently in a particle delivery with a certain average velocity $v$. Due to thermal activation the particle jumps over the potential barrier between neighboring fringes. ${ }^{27}$ Since the drag force of the medium acts against the particle motion, the periodic poten-

\footnotetext{
a)Electronic mail: siler@isibrno.cz.

${ }^{\text {b)} E l e c t r o n i c ~ m a i l: ~ t c 51 @ s t-a n d r e w s . a c . u k . ~}$

${ }^{c}$ Electronic mail: zemanek@isibrno.cz.
}

tial profile is tilted and the particle jumps more frequently over the lower potential barrier, i.e., the particle lags behind the moving fringes. ${ }^{6,28}$ Consequently, the delivery speed $v$ is lower compared to $u$. However, if multiple particles are transported together, their mutual optical and hydrodynamic interactions fix the inter-particle distances and suppress independent particle jumps over the potential barrier. The delivery speed of all the particles can, therefore, be significantly enhanced compared to that of a single particle.

We used the interference of two independent counterpropagating evanescent waves to create an array of optical traps near the surface ${ }^{4,6}$ (see Fig. 1). Two Gaussian beams were focused on the top surface of the prism where they formed overlapping spots $10 \mu \mathrm{m}$ wide and $100 \mu \mathrm{m}$ long with standing wave fringes separated by $200 \mathrm{~nm}$. We shifted the relative phase between both beams by a movable mirror $(\mathrm{MM})$ in a controlled fashion. Movement of the standing wave fringes was accompanied by a delivery of particles confined within the standing wave.

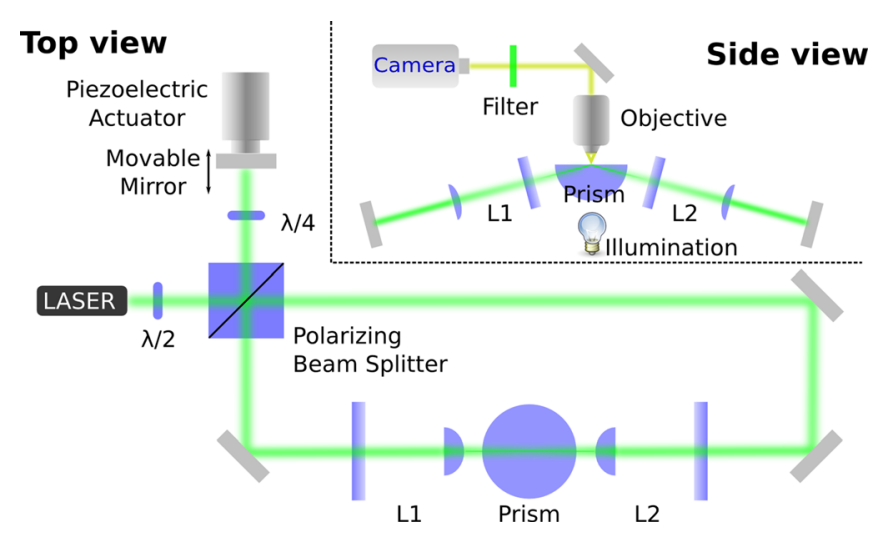

FIG. 1. (Color online) Experimental setup. Laser light (Coherent Verdi, $10 \mathrm{~W}, \lambda_{\text {vac }}=532 \mathrm{~nm}$ ) is separated into two beams by a polarizing beam splitter (PBS). One beam is reflected on the MM and returns to the PBS. Since the beam has passed twice through the quarter-wave plate $\lambda / 4$ its polarization is now perpendicular to the original one, thus it passes directly through the PBS and has the same polarization as the counter-propagating beam. Two pairs of cylindrical lenses L1, L2 focus the beams onto the upper surface of the BK7 prism in the form of a half-sphere lens (diameter $1 / 2^{\prime \prime}$ ) covered with a thin layer of immersion oil and a cover slip. MM was controlled by PIFOC (Physik Instrumente) which provided precise and fast movement over $50 \mu \mathrm{m}$ with velocity up to $200 \mu \mathrm{ms}^{-1}$. 

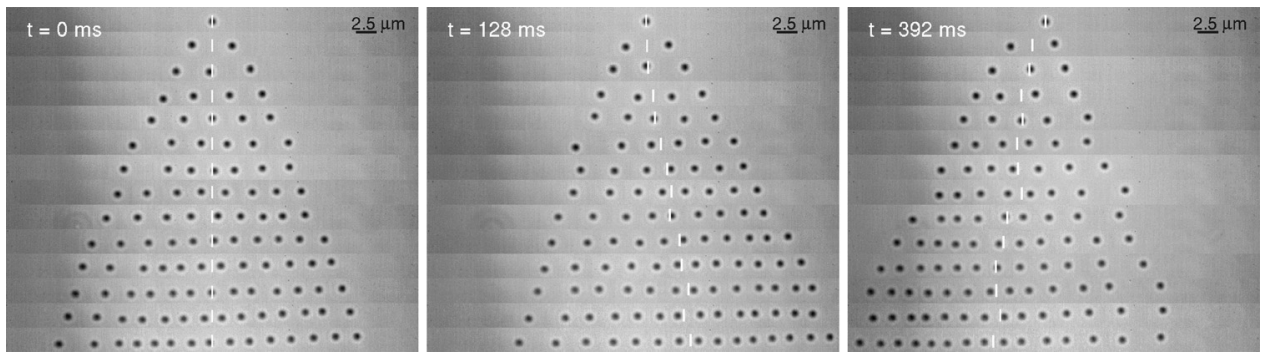

FIG. 2. Bright field images of chains formed from 1 to 15 polystyrene beads of diameter $520 \mathrm{~nm}$ placed in water and self-arranged in the evanescent standing wave. The images compare chain positions and particles locations inside each chain at three different time points. All chains are aligned in these pictures such that their centers of mass lie on the vertical dashed white line at $t=0$. The attached video shows the motion of all chains over one period of a sawtooth motion corresponding to $0.5 \mathrm{~s}$. Note that the chain made of 10 particles is missing because the 10th and 11th particles joined the chain at almost the same moment (enhanced online). [URL: http://dx.doi.org/10.1063/1.3680234.1]

The evanescent standing wave created a periodic array of optical traps (potential minima) separated by a potential barrier of height $\Delta U$ which depends not only on the incident laser power but also on the size of confined particles. ${ }^{6}$ Based on our previous results ${ }^{4,6}$ we used a water solution of polystyrene particles of diameter $520 \mathrm{~nm}$ because they form deep optical wells. The movement of particles was recorded by a fast charge coupled device (CCD) camera recording 250 frames per second. The MM performed a bidirectional sawtooth motion with velocity $76 \mu \mathrm{ms}^{-1}$. During the experiment additional particles entered the standing wave and consequently were confined and self-arranged in a particle chain. Within $4 \mathrm{~min}$ of recording up to 18 particles were selfarranged in the chain. Figure 2 compares the bidirectional motion of chains made up of different numbers of particles taken at three different times. Each horizontal stripe in the figure shows a chain formed from a constant number of particles that existed for a period of 2-50 s.

In the static standing wave a single particle is confined in an intensity maximum or minimum, depending on its size. It has recently been pointed out ${ }^{29}$ that the optical binding is able to tilt the periodic optical potential of the standing wave in such a way that due to the thermal activation the particles overcome the potential barrier between neighboring traps and self-arrange at inter-particle distances close to the case of counter-propagating but non-interfering waves. Once the standing wave starts to move, the particles follow its movement and the hydrodynamic drag force tilts the periodic potential profile of the standing wave. In the case of a single trapped particle it increases the probability of particle jumps to optical traps laying in the opposite direction to the particle's movement. ${ }^{6}$ The average speed of a single particle ${ }^{28}$ can be expressed as

$$
v=u-\frac{L k_{B} T\left[\exp \left(\gamma u L / k_{B} T\right)-1\right]}{\gamma \int_{0}^{L} \mathrm{~d} x \int_{x}^{x+L} \mathrm{~d} y \exp \left[\frac{U(y)-U(x)+(y-x) \gamma u}{k_{B} T}\right]},
$$

where $\gamma$ is Stokes drag coefficient, $k_{B}$ Boltzmann constant, $T$ temperature, and $U(z)=-\Delta U / 2 \cos (2 \pi z / L)$ is the potential energy profile created by the static standing wave ( $L$ is the distance between two neighboring interference fringes).

In principle, the same happens if there are more particles in the chain. However, as Fig. 2 demonstrates, the overall velocity of the particle chain increases with the number of particles. Figure 2 and the attached video show that as the chain moves in one direction, the particles in the center of the chain catch up with the leading particles and leave the tail particles behind. When the direction of the standing wave motion is reversed, the central particles again move faster and catch up with the leading ones. However, the particles never touch or collide with one another. Obviously, the number of particles jumps increases in the direction opposite to the standing wave motion. Moreover, Fig. 3(a) demonstrates that the particles in the middle of the chain move with the highest velocity because the presence of their neighboring particles suppresses the jumps over the potential barrier. Similar behavior was described for three particles moving along a circular fringe of the Bessel beam of higher order. ${ }^{20}$ Figure 3(b) shows the average speed of particles in the chain as the chain length increases.

Let us estimate the contributions of the hydrodynamic and optical interactions between particles to the speed enhancement at least to the lowest approximation. From the experiment we were able to determine the position of each particle at given time and consequently the corresponding
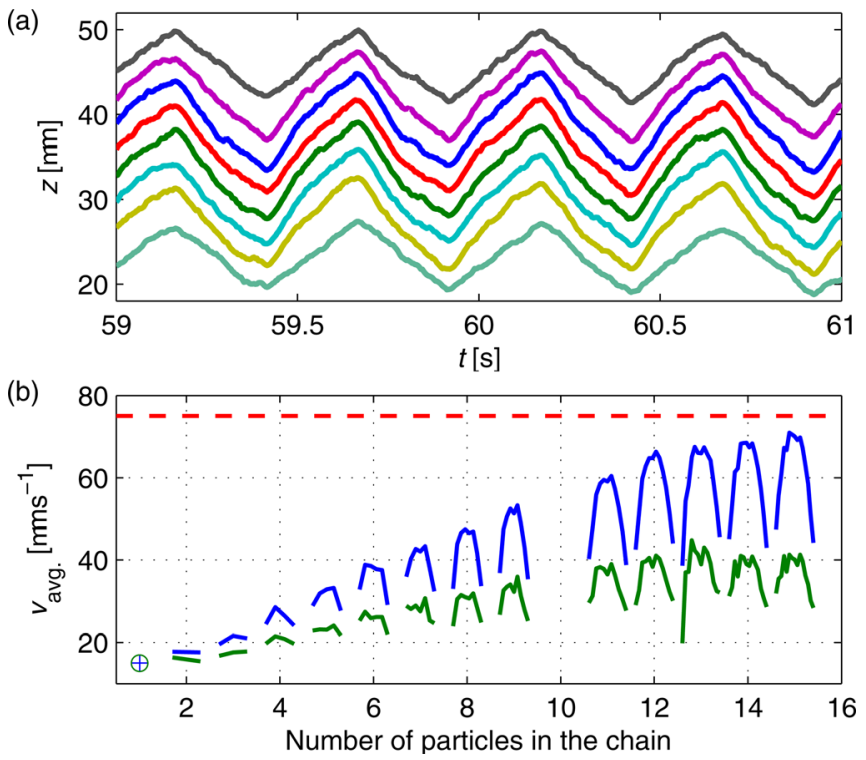

FIG. 3. (Color online) (a) An example of recorded positions of 8 particles in the chain. (b) Average velocities $v$ of individual particles in chains of increasing length. Each blue curve (upper curves) represents a single chain of a given number of particles. Green curves (lower ones) represent average particle velocity $\hat{v}$ if the hydrodynamic interaction is removed using the Oseen tensor. The dashed line shows the velocity $u$ of the moving interference fringes. 


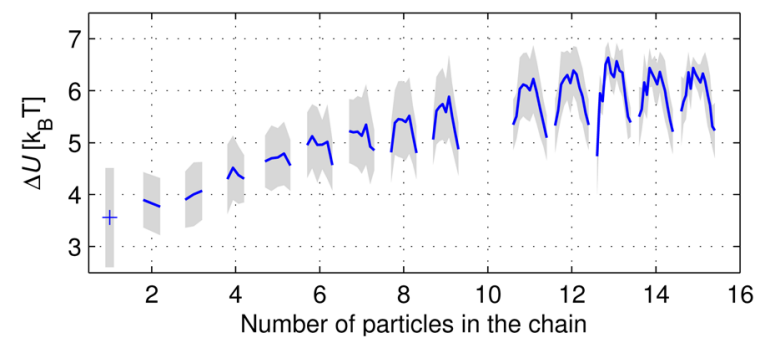

FIG. 4. (Color online) Change of $\Delta U$ with increasing number of particles in the chain. Each group of line segments represents a single chain of a given number of particles. Gray regions denote the error in the mean value.

velocity of each particle. As explained in Refs. 17 and 18, the Oseen tensor $\hat{H}_{i j}$ can be employed to express the effective force acting on the $i$-th particle without the hydrodynamic influence of other particles as $F_{i, \text { eff }}=\hat{H}_{i j}^{-1} v_{j}$, where $v_{j}$ is the measured speed of the $j$-th particle. This allows us to recalculate a reduced effective velocity $\hat{v}_{i}=F_{i, \text { eff }} / \gamma$ corresponding to the motion of a single isolated particle under the influence of the same optical field, thus decoupling lightmediated inter-particle interactions from hydrodynamic ones. Figure 3 compares average particle velocities $v_{i}$ and $\hat{v}_{i}$. We see that while the average speed of a single particle is $12.5 \mu \mathrm{ms}^{-1}$, the velocities $v_{i}\left(\hat{v}_{i}\right)$ of particles in the 15-particles chain vary from $71 \mu \mathrm{ms}^{-1}\left(41 \mu \mathrm{ms}^{-1}\right)$ in the chain center to $44 \mu \mathrm{ms}^{-1}\left(28 \mu \mathrm{ms}^{-1}\right)$ on its edges. Therefore, the hydrodynamic coupling contributes to the observed speed enhancement by more than $50 \%$; however, the remaining contribution signifies important optically mediated interactions within the system.

Since the velocity $\hat{v}_{i}$ can be considered equivalent to $v$ (Eq. (1)), it can be used to quantify the potential barrier $\Delta U_{N, i}$ associated with the $i$-th particle in a chain formed by $N$ particles. The results of this procedure are shown in Fig. 4. Comparing the optical trap depth of a single particle $\Delta U_{1,1}=3.8 k_{B} T$ and a central particle in a 15 -particle chain $\Delta U_{8,15}=6.4 k_{B} T$ we find a $68 \%$ increase due to the optical interaction between the particles. Unfortunately, the optical intensity of the trapping beams was inhomogeneous in the range of tens of percent over the region where the particles moved and this caused relatively large errors in $\Delta U_{N, i}$ (gray regions in Fig. 4). At this stage we did not take into account the surface proximity because it was found recently ${ }^{6,27}$ that the drag coefficient increase due to the surface proximity is almost compensated by the decrease of water viscosity due to localized heating by the trapping laser power.

In conclusion we demonstrated that optically selfarranged linear chains of particles move faster in a traveling standing wave with increasing number of particles. However, this increase tends to saturate for more than 13 particles and the observed speed increased about $6 \times$ compared to a single particle. Such an increase of the delivery speed of tens of self-arranged particles is useful for faster samples delivery in microfluidic systems. We deduce that two effects are respon- sible for the observed behavior. The hydrodynamic coupling between the particles reduces the viscous drag, and the optical binding increases the height of the potential barriers between the neighboring optical traps created by the standing wave fringes. Purely from the dynamics of the chains' motion we have been able to quantify the optical binding effects, and furthermore we have shown that the relative influence of both optical binding and hydrodynamic coupling is approximately of the same order.

The authors are obliged to Dr. Roberto Di Leonardo and Dr. Heather Dalgarno for their useful comments and acknowledge support from CSF (202/09/0348), MEYS CR (LC06007), and EC (ALISI No. CZ.1.05/2.1.00/01.0017).

${ }^{1}$ A. Ashkin, J. M. Dziedzic, J. E. Bjorkholm, and S. Chu, Opt. Lett. 11, 288 (1986).

${ }^{2}$ M. P. MacDonald, G. C. Spalding, and K. Dholakia, Nature 426, 421 (2003).

${ }^{3}$ K. Dholakia, M. P. MacDonald, P. Zemánek, and T. Čižmár, Methods Cell Biol. 82, 467 (2007).

${ }^{4}$ T. Čižmár, M. Šiler, M. Šerý, P. Zemánek, V. Garcés-Chávez, and K. Dholakia, Phys. Rev. B 74, 035105 (2006).

${ }^{5}$ P. Jákl, T. Čižmár, M. Šerý, and P. Zemánek, Appl. Phys. Lett. 92, 161110 (2008).

${ }^{6}$ M. Šiler, T. Čižmár, A. Jonáš, and P. Zemánek, New J. Phys. 10, 113010 (2008).

${ }^{7}$ K. Dholakia and P. Zemánek, Rev. Mod. Phys. 82, 1767 (2010).

${ }^{8}$ M. M. Burns, J.-M. Fournier, and J. A. Golovchenko, Phys. Rev. Lett. 63, 1233 (1989).

${ }^{9}$ S. A. Tatarkova, A. E. Carruthers, and K. Dholakia, Phys. Rev. Lett. 89, 283901 (2002).

${ }^{10}$ V. Garcés-Chávez, K. Dholakia, and G. C. Spalding, Appl. Phys. Lett. 86, 031106 (2005).

${ }^{11}$ C. D. Mellor and C. D. Bain, Chem. Phys. Chem. 7, 329 (2006).

${ }^{12}$ P. J. Reece, V. Garcés-Chávez, and K. Dholakia, Appl. Phys. Lett. 88, 221116 (2006).

${ }^{13}$ O. Brzobohatý, V. Karásek, T. Čižmár, and P. Zemánek, Appl. Phys. Lett. 99, 101105 (2011).

${ }^{14}$ J. Happel and H. Brenner, Low Reynolds Number Hydrodynamics (Prentice-Hall, Englewood Cliffs, 1965).

${ }^{15}$ M. Polin, D. G. Grier, and S. R. Quake, Phys. Rev. Lett. 96, 088101 (2006).

${ }^{16}$ A. M. Yao, S. A. J. Keen, D. R. Burnham, J. Leach, R. D. Leonardo, D. McGloin, and M. J. Padgett, New J. Phys. 11, 053007 (2009).

${ }^{17}$ R. Di Leonardo, S. Keen, J. Leach, C. D. Saunter, G. D. Love, G. Ruocco, and M. J. Padgett, Phys. Rev. E 76, 061402 (2007).

${ }^{18}$ A. Curran, A. Yao, G. Gibson, R. Bowman, J. Cooper, and M. Padgett, J. Biophotonics 3, 244 (2010).

${ }^{19}$ J. E. Curtis and D. G. Grier, Phys. Rev. Lett. 90, 133901 (2003).

${ }^{20}$ M. Reichert and H. Stark, J. Phys.: Condens. Matter 16, S4085 (2004).

${ }^{21}$ B. S. Schmidt, A. H. J. Yang, D. Erickson, and M. Lipson, Opt. Express 15, 14322 (2007).

${ }^{22}$ T. Čižmár, V. Garcés-Chávez, K. Dholakia, and P. Zemánek, Appl. Phys. Lett. 86, 174101 (2005).

${ }^{23}$ D. M. Gherardi, A. E. Carruthers, T. Čižmár, E. M. Wright, and K. Dholakia, Appl. Phys. Lett. 93, 041110 (2008).

${ }^{24}$ T. Čižmár, O. Brzobohatý, K. Dholakia, and P. Zemánek, Las. Phys. Lett. 8, 50 (2011)

${ }^{25}$ K. Grujic and O. G. Hellesø, Opt. Express 15, 6470 (2007).

${ }^{26}$ M. Šiler, T. Čižmár, M. Šerý, and P. Zemánek, Appl. Phys. B 84, 157 (2006).

${ }^{27}$ M. Šiler and P. Zemánek, New J. Phys. 12, 083001 (2010).

${ }^{28}$ P. Reimann, Phys. Rep. 361, 57 (2002).

${ }^{29}$ O. Brzobohatý, V. Karásek, M. Šiler, J. Trojek, and P. Zemánek, Opt. Express 19, 19613 (2011). 